\title{
QUANTIFICAÇÃO E GEORREFERENCIAMENTO SEMIAUTOMÁTICO DE ÁRVORES URBANAS
}

\author{
QUANTIFICATION AND SEMI-AUTOMATIC GEOREFERENCING OF URBAN TREES
}

Ricardo Luís Barbosaํ, Rodrigo Bezerra de Araújo Gallis², Alan Kazuo Hiraga ${ }^{3}$, Francisco Assis da Silva ${ }^{4}$

\section{RESUMO}

O inventário de árvores urbanas é importante para o conhecimento das espécies existentes e a geolocalização contribui para uma gestão eficiente. Diversas técnicas para a realização de tal inventário são apresentadas na literatura e a produtividade e desempenho são os mais diversos possíveis. Muitos trabalhos na literatura nacional realizam uma amostragem para fazer inferência sobre a população das espécies em um município, para se reduzir o tempo de levantamento e consequentemente os custos. Nesse trabalho, é apresentada uma metodologia inovadora para a produção de dados para a realização do inventário, que consiste em uma unidade de mapeamento móvel, um conjunto de câmaras e sensores GNSS para o georreferenciamento semiautomático dos indivíduos presentes nas ruas e avenidas da mancha urbana da cidade de Monte Carmelo - MG. Todos os indivíduos presentes nas vias foram georreferenciados, totalizando 7337 árvores e em um tempo de produção (aproximadamente 100 horas) consideravelmente baixo, quando comparado com outras técnicas de levantamento. Em trabalhos futuros, pretende-se cadastrar as espécies, altura, condições de saúde e disponibilizar os dados em um sigweb.

Palavras-chave: Mapeamento móvel; Sistema de Informação Geográfica; Geolocalização de árvores.

\section{ABSTRACT}

The inventory of urban trees is important to acknowledge existing species and geolocation contributes to efficient management. Several techniques to perform an inventory are presented in the literature and productivity and performance are as diverse as possible. Many studies in the national literature perform a sampling to make inferences on the species' population in a municipality level, which reduces the time spent for data survey and consequently the costs. In this paper, an innovative methodology is presented to produce data for inventories, which consists of a mobile mapping system, cameras and GNSS sensors for the semi-automatic georeferencing of the trees present on the streets and avenues of the urban environment of the city of Monte Carmelo - MG. All the individuals present in the streets were georeferenced, totaling 7377 trees and a considerably low production time (approximately 100 hours) when compared to other survey techniques. In future studies, registration about the species, height, and health conditions of the trees is intended, and the data shared in a webgis.

Keywords: Mobile mapping; Geographic Information System; Trees Geolocation.

\section{INTRODUÇÃO}

Recebido em 19.02.2019 e aceito em 02.04.2019

1 Matemático. Doutor. Professor da Universidade Federal de Uberlândia - UFU. Monte Carmelo/MG. E-mail: rluisbarbosa@ufu.br

2 Engenheiro Cartógrafo. Doutor. Professor da Universidade Federal de Uberlândia - UFU. Monte Carmelo/MG. E-mail: rodrigogallis@ufu.br

3 Cientista da Computação. Mestre. Diretor da Integral Soluções. Presidente Prudente/SP. Email: alan@integralsol.com.br

4 Cientista da Computação. Doutor. Professor da Universidade do Oeste Paulista - UNOESTE. Presidente Prudente/SP. E-mail: chico@unoeste.br 
Um inventário florestal urbano possibilita uma gestão mais eficiente dos recursos naturais presentes na cena e também um melhor gerenciamento, pois, com o conhecimento das espécies e as suas condições, é possível fazer um planejamento de podas, identificar áreas de risco de quedas, plantio de árvores em áreas deficitárias, estimar os benefícios da manutenção e ampliação da quantidade de indivíduos (GRISE; BIONDI; ARAKI, 2016).

A presença de árvores urbanas contribui para a redução do ruído de trânsito, produção de sombra, redução da temperatura, sequestro de carbono, bem estar social, qualidade de vida dos moradores e visitantes, dentre outros (BARBEDO et al., 2005; MELAZO; NISHIYAMA, 2010; FERRAZ, 2012).

Por outro lado, a gestão municipal deve ser eficiente para prevenir quedas de árvores e de galhos que prejudicam a rede elétrica e podem produzir danos em automóveis e em pedestres, de galhos que reduzem a iluminação pública noturna, de raízes que estragam as calçadas, entre outros.

O levantamento dos dados para um inventário da arborização urbana pode ser realizado de várias maneiras e com custos variados, desde levantamento com sistemas que utilizam tecnologia de varredura laser terrestre, levantamento topográfico, levantamento com dispositivos móveis (celular, GPS de navegação), imagens de satélite entre outros (HOLOPAINEN et al., 2011; BORGES; MARIM; RODRIGUES, 2012).

Algumas técnicas utilizadas para a redução de custos é realizar uma amostragem espacial ou realizar um inventário em uma pequena área que podem dar uma estimativa dos indivíduos presentes na cidade (SILVA et al., 2016).

Uma opção mais recente é o uso de Veículos Aéreos Não Tripulados (VANT) que podem cobrir uma área relativamente grande com um baixo custo quando comparado com o levantamento aéreo tradicional, e podem oferecer alta resolução espacial, mas também, assim como as imagens de aerolevantamento e imagens de satélite, não conseguem discriminar os indivíduos de forma satisfatória.

Um sistema desenvolvido pelo Instituto de Pesquisas Tecnológicas (Arbio), cujos dados são coletados com smartphone, pode ser utilizado para gestão da arborização urbana, com funcionalidade de cadastro, vistoria e organização fitogeográfica (VERÍSSIMO et al., 2013). Existem outros sistemas comerciais que fazem a gestão do inventário com uso de SIG (Sistema de Informação Geográfica), mas em todos eles, a aquisição dos dados é basicamente manual e depende do operador ir a campo para georreferenciar e obter dados e imagens dos indivíduos para fins de registro no banco de dados.

Alguns projetos que utilizam o Google Maps e Street View, como o REGISTREE (WEEGNER et al., 2016), detecta, georreferencia e extrai atributos de modo automático e foi 
testado na cidade de Pasadena, EUA. O sistema automaticamente identifica e reconhece a espécie, combinando imagens aéreas e terrestres. Consegue ainda estimar o diâmetro da altura do peito de cada espécie localizada nas vias e foi testado com uma base pública (prefeitura da cidade) com cerca de 80000 indivíduos e mostrou uma acurácia de cerca de 75\%. Outro trabalho que utiliza os dados do Google Street View para computar a área verde disponível para os pedestres realizou uma amostra aleatória para fazer o estudo (LI et al., 2015).

Muitas outras cidades disponibilizam esse tipo de informação e incentivam os cidadãos a participarem da gestão desse importante ativo ambiental. O mapeamento colaborativo permite uma ampliação do georreferenciamento e de informações mais detalhadas dos indivíduos, porém ainda é incipiente no Brasil, mas é possível estimular a demanda por políticas públicas através de organizações sociais.

Nesse trabalho foi testada uma metodologia semiautomática de georreferenciamento que pode auxiliar os municípios a realizarem o inventário das árvores urbanas e o mapeamento colaborativo pode ajudar na identificação das espécies e no gerenciamento, além de ser uma metodologia de baixo custo, visto que os sensores utilizados, tanto de posicionamento quanto de imageamento, possuem um preço de aquisição relativamente baixo em relação a outras tecnologias de levantamento utilizados em sistemas de mapeamento móvel, como sistemas de escaneamento a laser e câmaras panorâmicas esféricas, por exemplo.

\section{MATERIAL E MÉTODOS}

O Município de Monte Carmelo - MG tem aproximadamente 50 mil habitantes e área de $1.354 \mathrm{Km}^{2}$, com clima Cwa, subtropical continental segundo a classificação global de Köppen, ou seja, apresenta um inverno seco e um verão chuvoso, temperatura média de $21^{\circ}$ e cerca de $1400 \mathrm{~mm}$ de precipitação média anual. A cidade está localizada na mesorregião do Triângulo Mineiro e Alto Paranaíba. A vegetação nativa do município está inserida no bioma cerrado e com a urbanização do município foram inseridas espécies exóticas.

Um sistema de mapeamento móvel percorreu as ruas da cidade de Monte Carmelo-MG (mancha urbana) e coletou vídeos com resolução de 4K. O levantamento foi feito no mês de julho de 2016 e realizado por dois operadores, um para dirigir o automóvel e outro para controlar o sistema de captura e controlar a navegação. Foram percorridos cerca de $300 \mathrm{~km}$ dentro da mancha urbana do município, incluídos os deslocamentos para acessar as vias. A duração foi de aproximadamente 16 horas em dois dias de levantamento (BARBOSA et al., 2016). Neste levantamento foram coletados os dados utilizados na produção desse artigo. 
Na figura 1 é mostrada uma parte do sistema com as câmaras GoPro Black Hero 3.0. Esse modelo de câmara, denominado action cam, ou câmara de ação, foi escolhida para a composição do sistema pela robustez e bom funcionamento em situações adversas, como transitar com o veículo em ruas e estradas de terra.

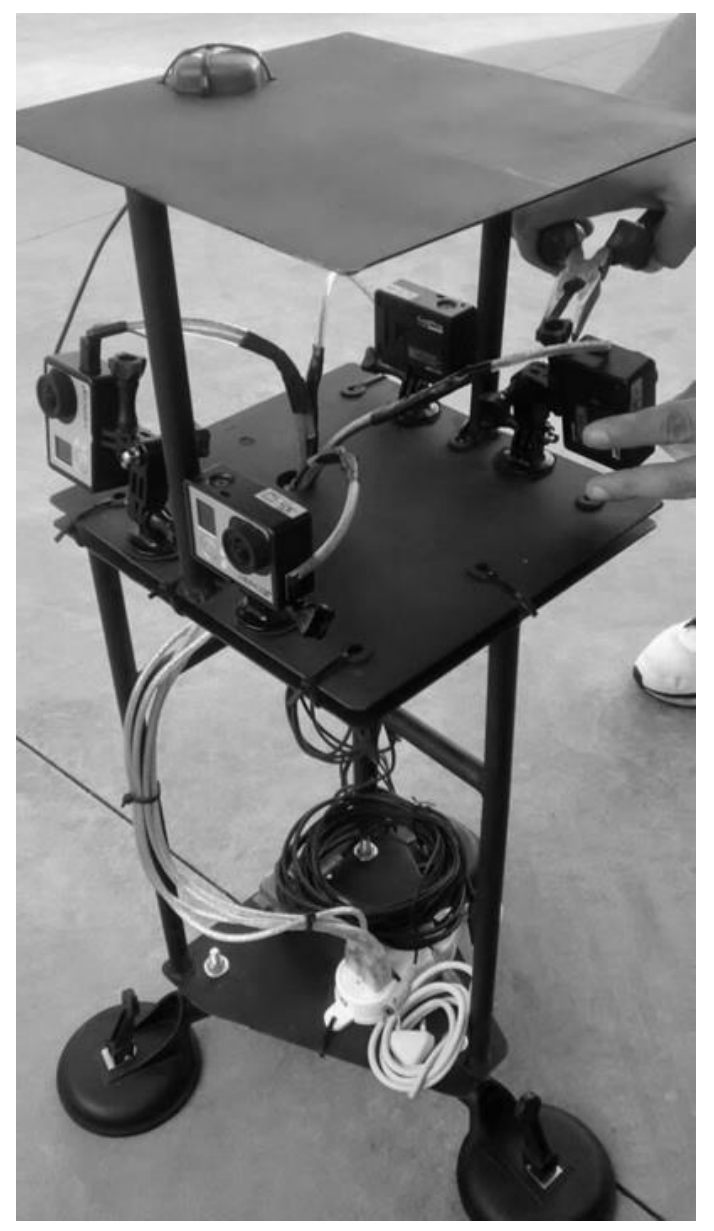

Figura 1. Configuração das câmaras GoPro Black Hero 3.0

Figure 1. GoPro Black Hero 3.0 Cameras configuration

Essa disposição das câmaras permite coletar os vídeos dos dois lados da via e a câmara rotacionada permite enquadrar objetos (árvores) com uma altura de um prédio de quatro andares. Integrado com as câmaras, existe um sensor GNSS (Global Navigation Satellite System) da marca Xsens (figura 2), que coleta dados de posicionamento e de orientação da câmara (ângulos de Euler). 


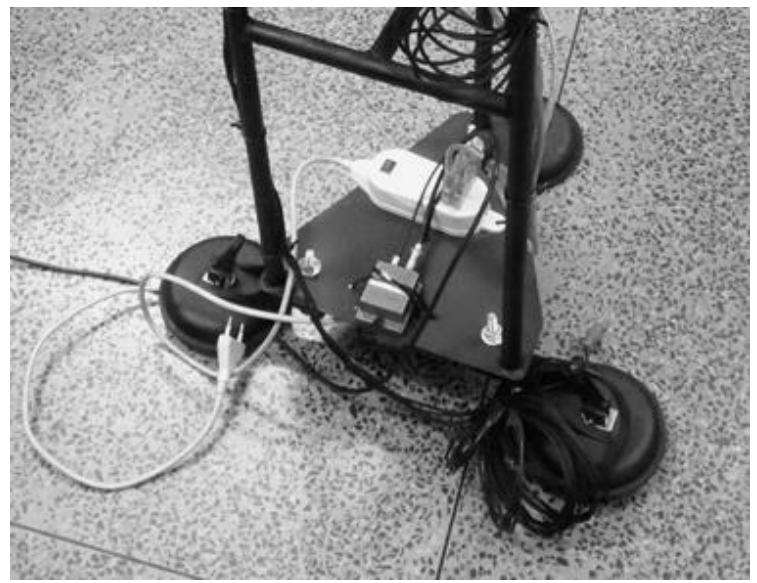

Figura 2. Sensor de posicionamento Xsens

Figure 2. Xsens positioning sensor

Antes de iniciar o levantamento, foi realizado o planejamento, onde todas as vias (ruas e avenidas) da cidade foram vetorizadas em um aplicativo implementado em C\#. A cidade foi dividida em 4 regiões e um algoritmo informa qual a melhor rota a ser seguida para otimizar o tempo de levantamento de modo análogo ao popularmente chamado GPS de automóvel.

Após o planejamento e com o sistema embarcado no automóvel, a posição é atualizada pelo sensor GNSS e é iniciado o processo de coleta (vídeos e as coordenadas da posição e orientação do sensor). As câmaras são ligadas ao mesmo tempo com um controle remoto, via wifi. Ao começar uma rota, as câmaras são ligadas e o vídeo é capturado, bem como o sinal GNSS. Ao finalizar o percurso, o operador encerra a rota e o sistema indica qual a próxima rota que é a melhor para reduzir o tempo do levantamento, e o processo se repete, até finalizar todas as rotas planejadas. Com o levantamento finalizado, os vídeos são transferidos da câmara (que armazena os vídeos em um cartão microSD) para um computador.

A Figura 3 ilustra a metodologia com o georreferenciamento das árvores já realizado. No lado esquerdo da figura estão as vias levantadas com um marcador de status se já foram processadas ou não. O operador escolhe uma via e o vídeo é reproduzido. Existem os botões para tocar o vídeo, retroceder, avançar e recortar a imagem do vídeo, além do botão para confirmar a operação (gravar as informações no banco de dados). O círculo branco representa a posição onde uma árvore será georreferenciada quando o operador clicar no ícone da tesoura (recorte) e em seguida confirmar a operação. 


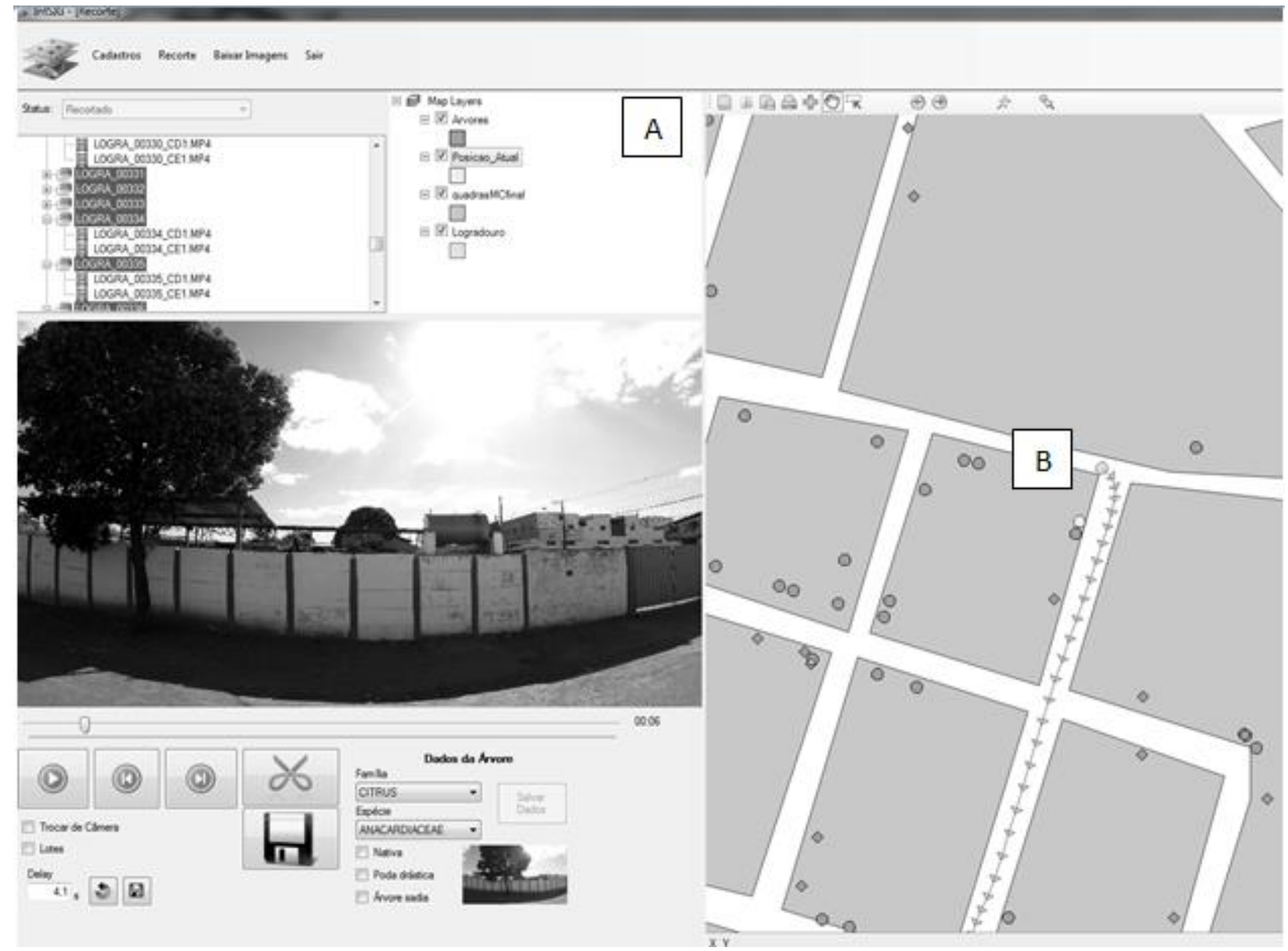

Figura 3. Exemplo da interface do software para o georreferenciamento das árvores ilustrando (A) a escolha de uma via a ser visualizada e os comandos para a edição do vídeo e (B) mostrando o sentido do deslocamento e a posição um momento antes do posicionamento de uma árvore (círculo branco)

Figure 3. Example of software interface for tree georeferencing illustrating $(A)$ the choice of a path to be viewed and the commands for video editing and (B) showing the direction of the movement and the position a moment before the positioning a tree (white circle)

Quando o operador enquadrar a árvore (o círculo branco está sobre o círculo mais escuro, que no sistema é verde), a árvore estará georreferenciada naquela posição (figura 4). 


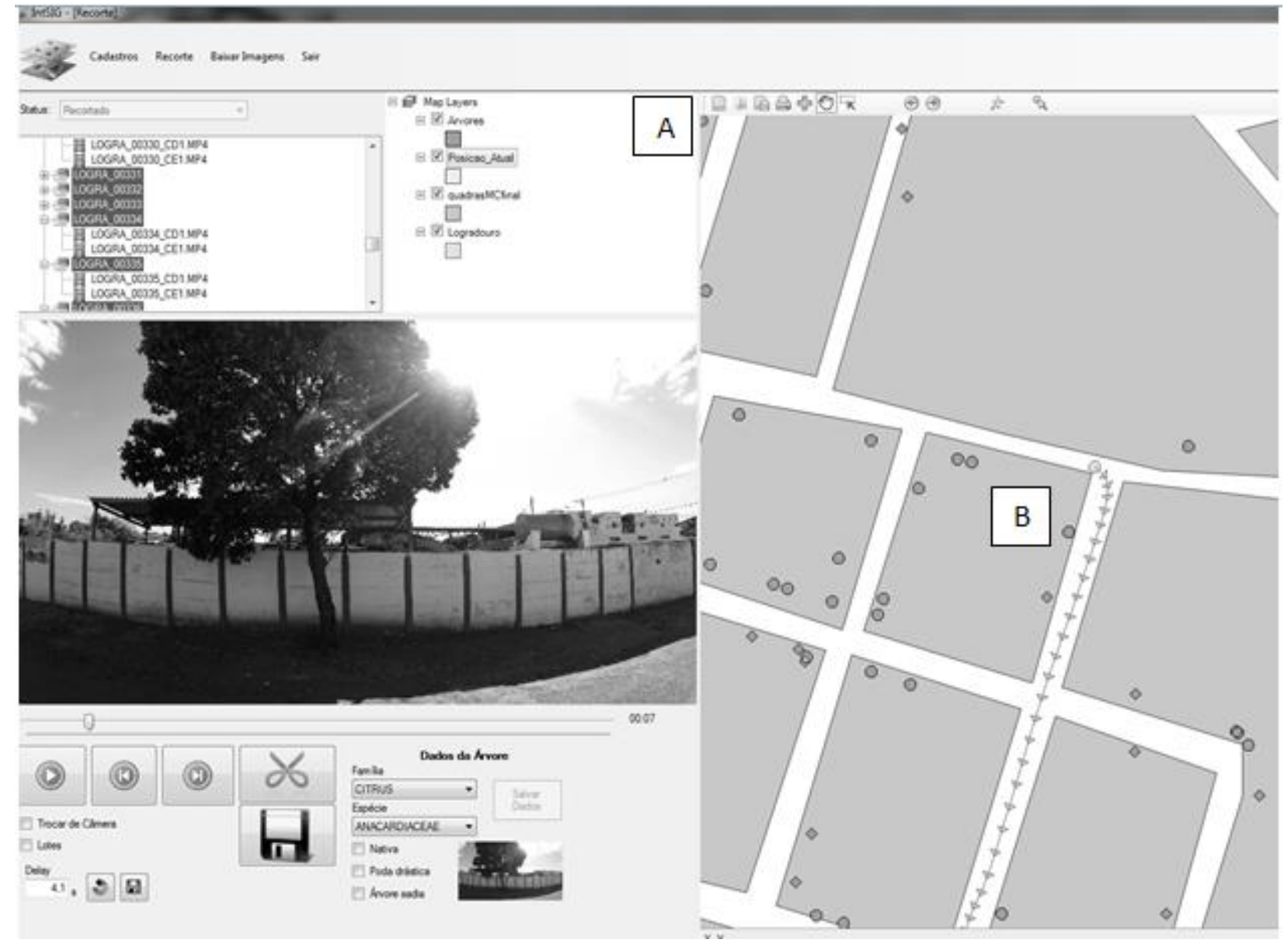

Figura 4. Após o recorte da imagem, o operador clica no ícone do disquete (A) e os dados são armazenados no banco de dados. Na figura (B), o círculo branco está sobre o círculo preenchido que representa a árvore georreferenciada

Figure 4. After cropping the image, the operator clicks the floppy disk icon $(A)$ and the data is stored in the database. In the figure (B), the white circle is over the filled circle that represents the tree georeferencing

O sistema é programado para colocar o círculo branco três metros de modo perpendicular ao sentido do deslocamento devido à largura padrão de uma via. A posição da coordenada da posição da câmara está indicada pela seta no eixo central da via. O primeiro círculo no centro da via indica onde começou a gravação do vídeo. O sistema também foi implementado em C\# com a biblioteca DotSpatial, com banco de dados MySQL.

Após a inspeção de todos os vídeos, tem-se as coordenadas das árvores que são exportadas em formato .txt e importadas pelo software QGis, onde foi realizado o processo de edição cartográfica e gerados os mapas. 


\section{RESULTADOS E DISCUSSÃO}

Foram percorridas 328 rotas, o que produziu 1312 arquivos de vídeo, com um espaço de 699GB. A qualidade do vídeo 4k gera uma imagem de alta resolução, que permite inclusive visualizar as folhas das árvores, o que auxilia na identificação da espécie, porém, para realizar o processo de recorte, é necessário reduzir a qualidade, pois o consumo de memória é muito grande, o que reduz a produtividade. A redução da qualidade dos vídeos demorou cerca de 4 horas.

Os dados GNSS foram processados por software proprietário e gerados os shapes para visualização no sistema de recorte. Os dados do sensor GNSS ocuparam aproximadamente $12 \mathrm{MB}$ de memória. Foram georreferenciadas 7337 árvores, cuja geolocalização são mostradas na figura 5.

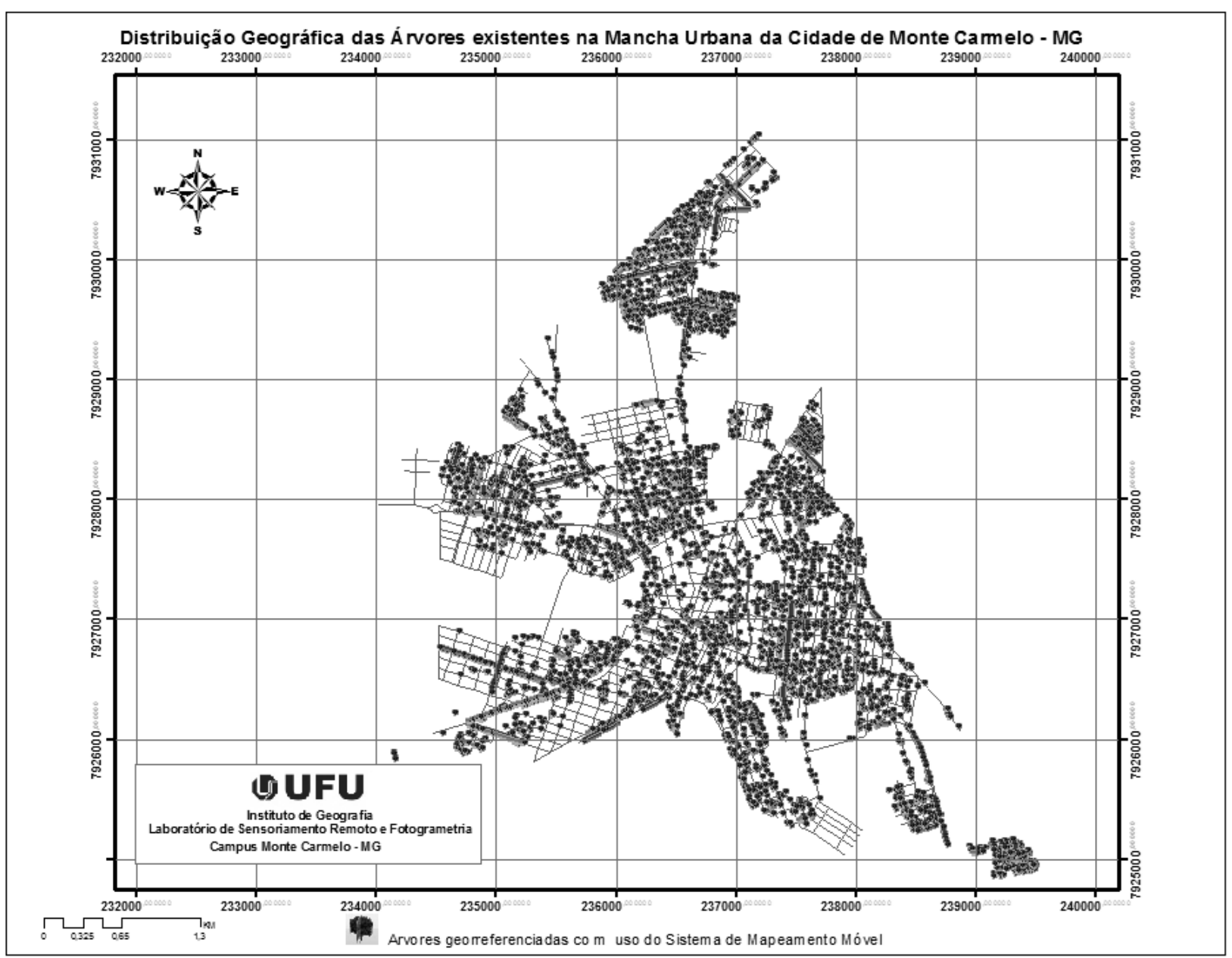

Figura 5. Mapa da espacialização das árvores urbanas de Monte Carmelo - MG Figure 5. Map of tree geolocalization in Monte Carmelo - MG

Outra visualização possível é usar o Google Maps, que auxilia na análise qualitativa do inventário dos indivíduos (Figura 6). 


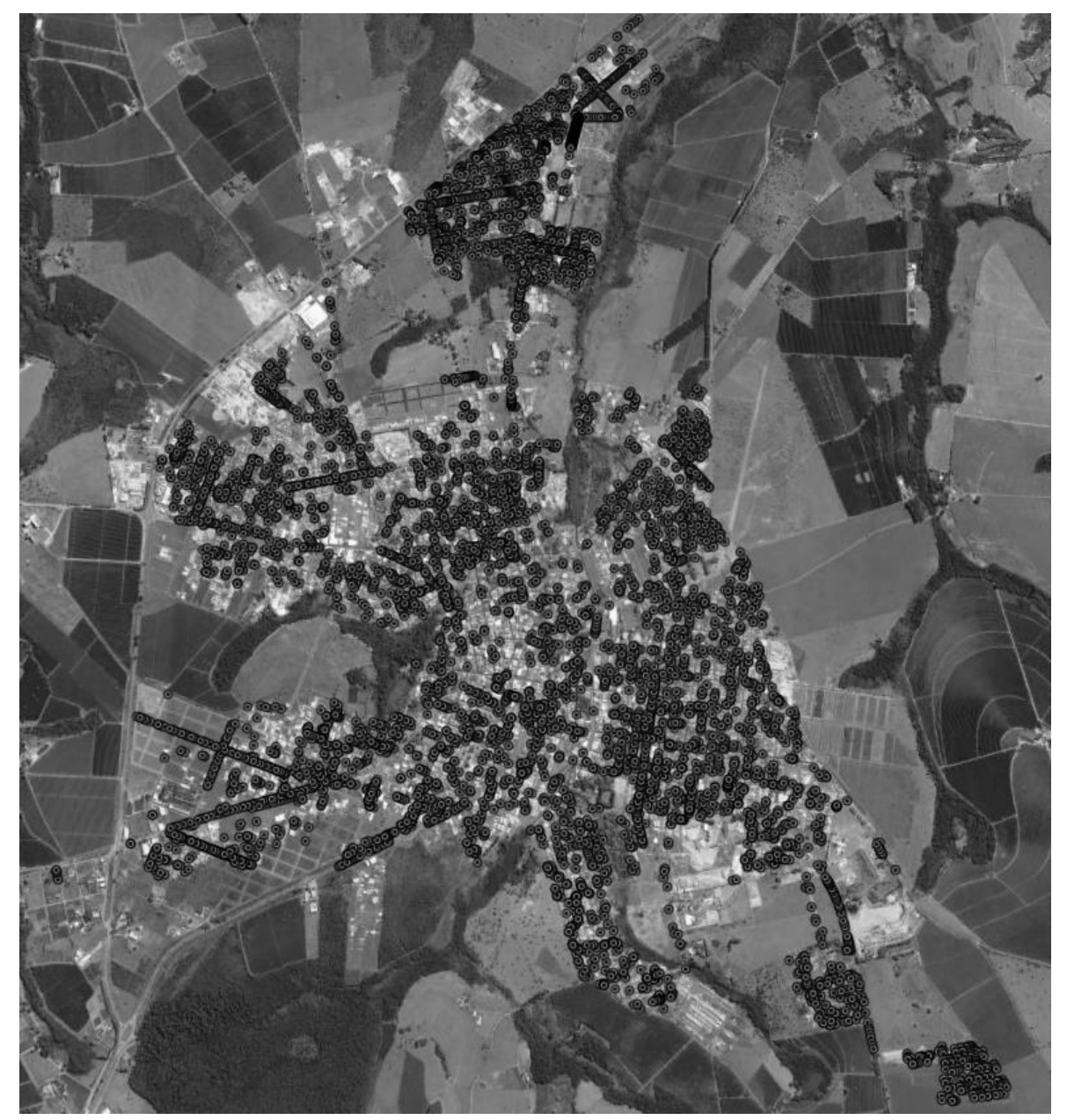

Figura 6. Geovisualização com a base do Google Maps

Figure 6. Geovisualization in Google Maps

Com a resolução $4 \mathrm{~K}$ é possível também o operador fazer um zoom na espécie para visualizar a folha e um especialista pode fazer a classificação da espécie em um trabalho de escritório e em dúvida, compartilhar a imagem com outros especialistas, botânicos, engenheiros florestais, engenheiros ambientais, agrônomos para certificar a espécie. Também é possível verificar as árvores que podem causar dados à rede elétrica, dentre outros conflitos com equipamentos urbanos (Figura 7). 


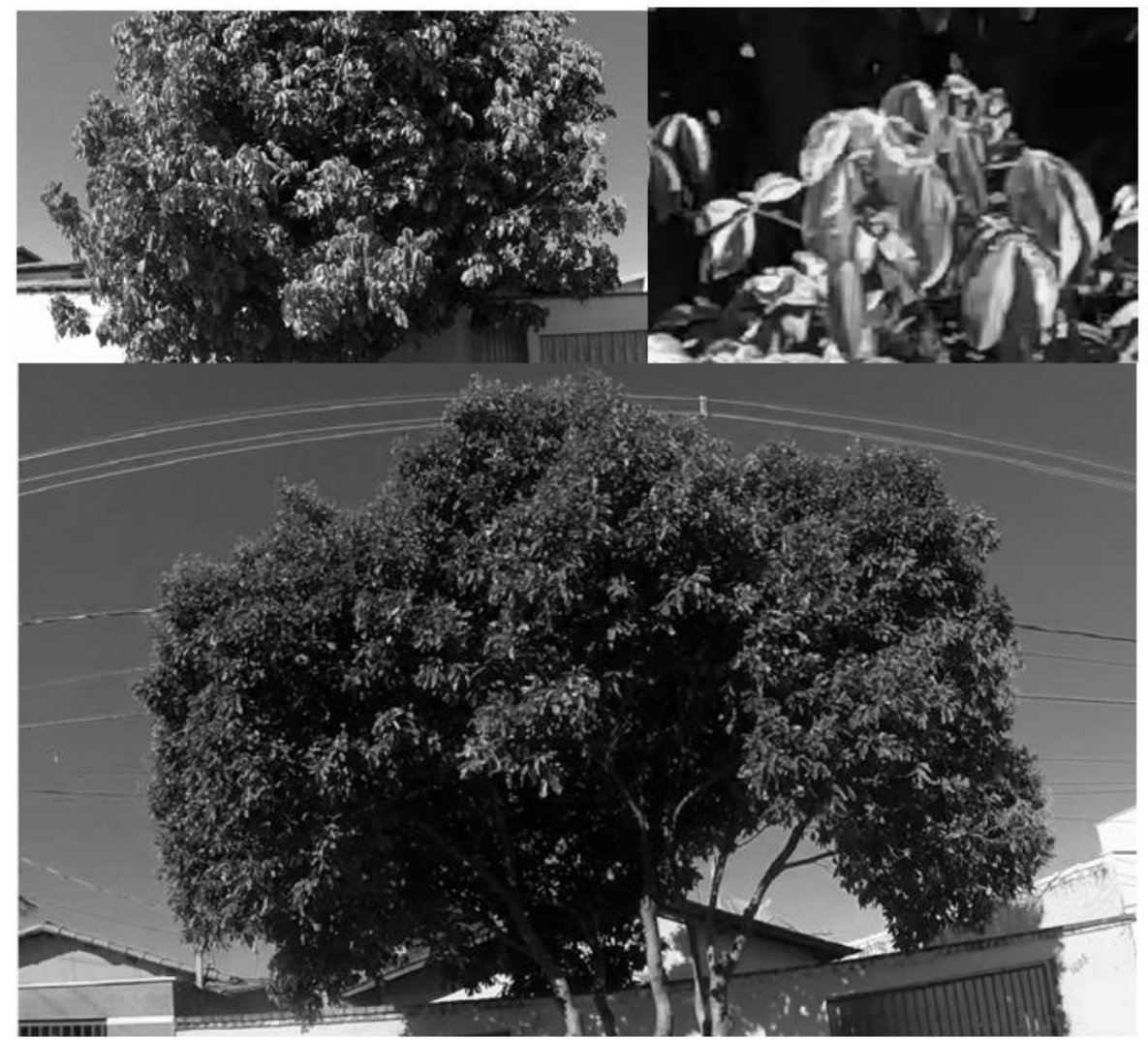

Figura 7. Imagem em resolução de 4k coletada com o sistema de mapeamento móvel Figure $7.4 \mathrm{k}$ resolution image collected by the mobile mapping system

A metodologia se mostrou eficaz em termo de aquisição dos dados, pois em 16 horas foi possível levantar os dados de toda a malha urbana, que pode ser facilmente aplicada em grande parte dos pequenos e médios municípios brasileiros. Em comparação com outras metodologias de levantamento de dados geoespaciais, como os levantamentos topográficos ou geodésicos, o caminhamento fotogramétrico onde os sensores de posicionamento e imageamento são embarcados em veículos automotores se mostram uma alternativa que fornece rapidez e precisão principalmente. A metodologia também se mostra segura, pois não há a necessidade do operador ou técnico descer do veículo para coletar informações acerca do objeto a ser medido (SILVA; CAMARGO; GALLIS, 2003, BARBOSA, 2006).

O trabalho de escritório para processar e produzir as informações geoespaciais levou cerca de 84 horas, o que torna a produção da informação espacial muito rápida e possibilita a realização do inventário florestal urbano completo de forma ágil. No caso da metodologia utilizada nesta pesquisa, em 100 horas de trabalho (84 horas de processamento e 16 de trabalho de campo) levantou-se um total de 7337 árvores, uma média de 73 árvores por hora aproximadamente. 
As imagens também podem ser utilizadas para se fazer um planejamento do manejo das árvores urbanas e a classificação poderá ajudar em propostas de replantio e espécies mais adequadas para a cidade.

Em Li et al. (2015) foram utilizadas imagens obtidas com o Google Street Map e uma ferramenta do Google para produzir um índice de vegetação com uma amostra de 258 mosaicos para inferir sobre o índice, mas essa mesma metodologia não pode ser aplicada em muitas cidades do interior do Brasil, onde muitas vezes não há cobertura das imagens do Street View, além da grande defasagem de tempo. Por exemplo, no município de Monte Carmelo as imagens existentes foram coletadas no ano de 2013.

Em diversos trabalhos que se procura realizar esse tipo de mapeamento, a dificuldade se encontra no levantamento dos dados, por exemplo, em Ferraz (2012) o levantamento de 558 espécies foram demorou dois meses, com 18 pessoas fazendo o trabalho de campo e ainda sem ter o georreferenciamento dos indivíduos.

Também com o uso de um automóvel para percorrer $420 \mathrm{~km}$ das vias da cidade de Itanhaém, os autores Maria, Biondi e Brobowski (2016) contaram 18128 espécies e depois, por amostragem, fizeram a análise qualitativa das espécies presentes na cena, mas não informaram o tempo gasto e nem se fizeram o georreferenciamento dos indivíduos.

A metodologia aqui apresentada tem a vantagem do tempo de levantamento de todos os indivíduos presentes nas vias de modo rápido, porém, são necessárias técnicas de visão computacional e fotogramétricas para que se possa automatizar o processo de georreferenciamento, medição dos atributos e classificação das espécies.

As etapas futuras consistem em se fazer a catalogação das espécies, com o apoio de um especialista nessa classificação. A medição do diâmetro da altura do peito também pode ser feita com técnicas de fotogrametria e visão computacional, visto que a orientação das câmaras está disponível. Para essas etapas, será necessário implementar um sistema onde o especialista abra as imagens e faça a classificação das espécies, além dos outros atributos e características de acordo com um banco de dados que será modelado para esse fim e, posteriormente, disponibilizado em uma aplicação Web.

\section{CONCLUSÕES}

Esse projeto contribui para a criação de uma metodologia rápida e precisa de levantamento de dados das árvores urbanas, que pode ser usada em outras áreas, como para fins de cadastramento de imóveis e outras feições presentes nas vias urbanas (ativo de iluminação pública, comércio, serviço) e também contribuir com o mapeamento sistemático da 
cidade. O mapeamento móvel, que como estado da arte, vem incorporando avançadas tecnologias, como as câmaras integradas de forma a gerar imagens georreferenciadas na coleta dados de forma rápida e acurada.

De um ponto de vista técnico, um acervo de imagens e dados de geolocalização das árvores situadas na mancha urbana das cidades ajudará, juntamente com outras informações, os administradores e pesquisadores a realizar um manejo adequado e eficiente.

A metodologia desenvolvida permite conhecer a quantidade exata de árvores na área urbana, a localização com precisão, as espécies predominantes, suas condições, tamanhos da copa e altura. Sem contar a possibilidade de estudos individuais da árvore através da observação de sua imagem.

\section{AGRADECIMENTOS}

Os autores agradecem ao CNPq pelo financiamento recebido № 483413/2012-7, a CAPES pela concessão de Bolsa de Estágio Sênior no Exterior (Processo 99999.002696/2015-00) e aos revisores pelas relevantes contribuições ao manuscrito.

\section{REFERÊNCIAS}

BARBEDO, A. S. C.; BIANCHI, C. G.; KELLER, L. R.; ORTEGA, M. G.; ORTEGA, S. E. H. Manual técnico de arborização urbana. 2.ed. São Paulo: PMSP-SVMA, 2005. 45p.

BARBOSA, R. L. Caminhamento fotogramétrico utilizand o o fluxo óptico filtrado. Presidente Prudente, 2006. 103f. Tese (Doutorado em Ciências Cartográficas) - Faculdade de Ciências e Tecnologia, Universidade Estadual Paulista, Presidente Prudente, 2006.

BARBOSA, R. L.; HIRAGA, A. K.; GALLIS, R. B. A.; SILVA, F. A. Implementação e prova de conceito de um sistema de mapeamento móvel. In: Simpósio Regional de Agrimensura e Cartografia, 2016, Monte Carmelo, MG.

BORGES, C. A. R. F.; MARIM, G. C.; RODRIGUES, J. E. C. Revista da Sociedade Brasileira de Arborização Urbana, Piracicaba, v.7, n. 4, p.16-22, 2012.

FERRAZ, M. V. Inventário das árvores urbanas da cidade de Registro-SP. Revista da Sociedade Brasileira de Arborização Urbana, Piracicaba, v.7, n.2, p.80-88, 2012.

GRISE, M. M.; BIONDI, D.; ARAKI, H. A paisagem da floresta urbana de Curitiba-PR. Revista da Sociedade Brasileira de Arborização Urbana, Piracicaba, v.11, n.3, p. 37-55, 2016.

HOLOPAINEN, M.; VASTARANTA, M.; KANKARE, V.; KANTOLA, T.; KAARTINEN, H.; KUKKO, A.; VAAJA, M.; HYYPPA, J.; HYYPPA, H. Mobile terrestrial laser scanning in urban tree inventory. In: SILVILASER, 2011, Hobart, Australia. Proceedings of Silvilaser, Hobart, Australia: University of Tasmania, 2011. p. 1-7. 
LI, X.; ZHANG, C.; LI, W.; RICARD, R.; MENG, Q.; ZHANG, W. Assessing street-level urban greenery using Google Street View and a modified green view index. Urban Forestry \& Urban Greening, Amsterdam, v.14, p. 675-685, 2015.

MARIA, T. R. B. C.; BIONDI, D.; BROBOWSKI, R. Inventário florístico quali-quantitativo das vias públicas de Itanhaém-SP. Revista da Sociedade Brasileira de Arborização Urbana, Piracicaba, v.11, n. 4, p.98-114, 2016.

MELAZO, G. C.; NISHIYAMA, L. Mapeamento da cobertura arbóreo-arbustiva em quatro bairros da cidade de Uberlândia-MG. Revista da Sociedade Brasileira de Arborização Urbana, Piracicaba, v.5, n.2, p. 52-66, 2010.

SILVA, J. F. C.; CAMARGO, P. O.; GALLIS, R. B. A. Development of a low-cost mobile mapping system: a South American experience. Photogrammetric Record, New Jersey, v. 18, n. 101, p.5-26, 2003.

SILVA, K. A. R.; LELES, P. S. S.; GIÁCOMO, R. G.; MENDONÇA, B. A. F. de. Diagnóstico e uso de geoprocessamento para manejo da arborização urbana do bairro centro da cidade do Rio de Janeiro - RJ. Revista da Sociedade Brasileira de Arborização Urbana, Piracicaba, v.11, n. 4, p. $98-114,2016$.

VERÍSSIMO, D. B.; RUSSO, M. C.; BRAZOLIN, S.; AMARAL, R. D. de A. M. ARBIO: sistema de gestão da arborização. In: CONFERÊNCIA IADIS IBERO-AMERICANA COMPUTAÇÃO APLICADA, 2013, São Leopoldo, RS.

WEGNER, J.D.; BRANSON, S.; HALL, D.; SCHINDLER, K.; PERONA, P. Cataloging Public Objects Using Aerial and Street-Level Images - Urban Trees, IEEE Conference on Computer Vision and Pattern Recognition (CVPR), Los Angeles, 6014-23, 2016. 